\title{
Microwave assisted efficient one-pot synthesis of 3,5,6-trisubstituted-1,2,4-triazines from fatty acid hydrazides under solvent-free conditions and their antimicrobial activity
}

\author{
Abdul Rauf, Shweta Sharma, and Saloni Gangal \\ Department of Chemistry, Aligarh Muslim University, Aligarh 202002, India \\ E-mail: abduloafchem@gmail.com
}

\begin{abstract}
Rapid and efficient solvent-free synthesis of 3,5,6-trisubstituted-1,2,4-triazines from fatty acid hydrazides under microwave irradiation is described. The one-pot synthesis on solid inorganic support provides the products in good yields. The newly synthesized compounds are screened for antimicrobial activity. The structural features of the synthesized 1,2,4-triazines were characterized by IR, ${ }^{1} \mathrm{H}$ NMR, ${ }^{13} \mathrm{C}$ NMR, mass and elemental analysis.
\end{abstract}

Keywords: Fatty acid hydrazides, microwave irradiation, 3,5,6-trisubstituted-1,2,4-triazines, antimicrobial activity

\section{Introduction}

Microwave assisted organic synthesis (MAOS) continues to affect synthetic chemistry significantly by enabling rapid, reproducible and scaleable chemistry development. ${ }^{1}$ The use of microwave irradiation is an established tool in organic synthesis for achieving better selectivity, rate enhancement and reduction of thermal degradation byproducts. ${ }^{2,3}$ However these procedures are practically limited as solvents in a microwave oven at elevated temperatures create high pressure, which may cause explosion. One of the advances to overcome this problem is inorganic $^{4,5}$ solid supported organic synthesis which attracts attention because of enhanced selectivity, milder reaction conditions and associated ease of manipulation. It also provides an opportunity to work with open vessels and enhanced possibility for scaling up reactions. ${ }^{6,7}$

Numerous reactions including condensations, cycloadditions, heterocycle formation, and metal-catalyzed cross-coupling have been explored under microwave conditions, some of which have been applied to medicinal chemistry and total synthesis of natural products. ${ }^{8}$ MAOS can facilitate the discovery of new reactions and reduce cycle time in optimization of reactions. In addition it serves to expand chemical space in compound library synthesis. Moreover it is an 
acknowledged quick alternative and green technology in synthetic organic chemistry that also provides easier work-up procedures.

1,2,4-Triazines are a representative class of heterocyclic compounds with a wide variety of interesting properties which are used in medicine and agriculture. ${ }^{9-12}$ It has been associated with diverse pharmacological activities such as hypertension and inhibition of platelets, ${ }^{13}$ antileukemic, ${ }^{14}$ antiinflammatory ${ }^{15}$ and potent neuroprotective agents. ${ }^{16}$ The 1,2,4-triazine moiety is a structural element in antimalarial, ${ }^{17}$ anticancer, ${ }^{18}$ antifungal, ${ }^{19}$ anticonvulsant, ${ }^{20}$

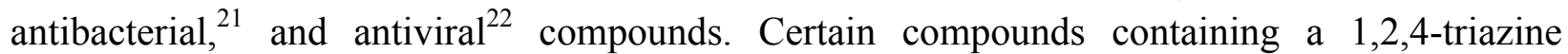
nucleus have been reported to possess pesticidal, ${ }^{23}$ neuropharmacological, ${ }^{24}$ analgesic and antidepressant $^{25}$ properties. Some 1,2,4-triazine derivatives are used for the determination of metal ions and as dyes. ${ }^{13} \mathrm{~N}$-Methyl derivatives of 1,2,4-triazines are the naturally occurring antibiotics fervenulin (planomycin), toxoflavin (xanthothricin) and reumycin.

Various methods for the synthesis of 1,2,4-triazines have been reported such as condensation of acid hydrazides with benzil in acetic acid containing $\mathrm{NH}_{4} \mathrm{OAc}$ to give 5,6- diphenyl-1,2,4triazine with various aromatic and heterocyclic groups attached at position $3,{ }^{26}$ cyclization of aliphatic, aromatic and heterocyclic acid hydrazides with 1,2-diketone mono-aroylhydrazones in alcoholic ammonia under pressure, ${ }^{27,28}$ condensation of monohydrazones of aromatic (but not aliphatic) 1,2-diketones with formation of novel 5,6-disubstituted-1,2,4-triazines, ${ }^{29}$ condensation of acid hydrazides and $\alpha$-halo acetophenone in the presence of $\mathrm{NaOAc}$ or AgOAc, ${ }^{30,31}$ reaction of $\alpha$-diketones with amidrazones and $S$-methylthiosemicarbazide, ${ }^{32-34}$ and condensation of acid hydrazides with diketones to afford 1,2,4-triazines under microwave irradiation. ${ }^{35}$

In view of above mentioned pharmacological importance of heterocycles and our ongoing efforts to synthesize heterocycles from fatty acids ${ }^{36-39}$ we now describe an expeditious ecofriendly solvent-free microwave accelerated solid state approach for the rapid synthesis of 3,5,6-trisubstituted-1,2,4-triazines from saturated and olefinic fatty acid hydrazides wherein several disadvantages such as long reaction time and tedious work-up are avoided.

\section{Results and Discussion}

We now report the synthesis of 3,5,6-trisubstituted-1,2,4-triazines 3a-I by condensation of 1,2diketonse 2a-b with various saturated and olefinic (internal and terminal) fatty acid hydrazides 1a-f under MW and solvent-free conditions in short reaction times (Scheme 1).

As can been seen from Tables 1 and 2, the scope of the reaction using saturated, olefinic (internal and terminal) and hydroxy fatty acid hydrazides was found to be good. 


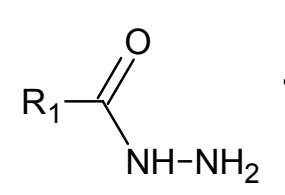

1a-f

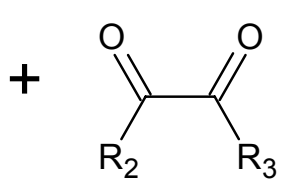

2a-b

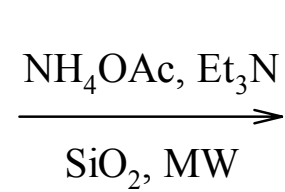

(a) $\mathrm{R}_{2}=\mathrm{R}_{3}=\mathrm{CH}_{3}$

(b) $\mathrm{R}_{2}=\mathrm{R}_{3}=\mathrm{Ph}$

Scheme 1<smiles>[R]c1nnc([R])c([R2])n1</smiles>

3a-1

Table 1. 3,5,6-Trisubstituted-1,2,4-triazines

\begin{tabular}{|c|c|c|c|c|}
\hline Entry & Starting from & $\mathbf{R}_{1}$ & $\mathbf{R}_{\mathbf{2}}$ & $\mathbf{R}_{3}$ \\
\hline 1 & $1 \mathbf{a}, 3 \mathbf{a}$ & & $\mathrm{CH}_{3}$ & $\mathrm{CH}_{3}$ \\
\hline 2 & $1 b, 3 b$ & & $\mathrm{CH}_{3}$ & $\mathrm{CH}_{3}$ \\
\hline 3 & $1 c, 3 c$ & & $\mathrm{CH}_{3}$ & $\mathrm{CH}_{3}$ \\
\hline 4 & 1d,3d & & $\mathrm{CH}_{3}$ & $\mathrm{CH}_{3}$ \\
\hline 5 & $1 e, 3 e$ & & $\mathrm{CH}_{3}$ & $\mathrm{CH}_{3}$ \\
\hline 6 & $\mathbf{1 f , 3 f}$ & & $\mathrm{CH}_{3}$ & $\mathrm{Ph}$ \\
\hline 7 & $1 \mathrm{a}, 3 \mathrm{~g}$ & & $\mathrm{Ph}$ & $\mathrm{Ph}$ \\
\hline 8 & $1 b, 3 h$ & & $\mathrm{Ph}$ & $\mathrm{Ph}$ \\
\hline 9 & $\mathbf{1 c , 3 i}$ & & $\mathrm{Ph}$ & $\mathrm{Ph}$ \\
\hline 10 & $\mathbf{1 d}, \mathbf{3 j}$ & & $\mathrm{Ph}$ & $\mathrm{Ph}$ \\
\hline 11 & $1 e, 3 k$ & & $\mathrm{Ph}$ & $\mathrm{Ph}$ \\
\hline 12 & 1f,3l & & $\mathrm{Ph}$ & $\mathrm{Ph}$ \\
\hline
\end{tabular}


The yield of 3,5,6-trisubstituted-1,2,4-triazines did not depend on the length of the fatty acid chain hydrazide Table 2. In order to determine the optimum conditions for the synthesis of triazines, variations in molar ratios of reagents and the irradiation time and power level of microwave set-up were investigated. After some experimentation, we found a set of conditions that generally provides products in good yield. The optimum conditions employed are that the molar ratio of fatty acid hydrazide and 1,2-diketone is 1:1 and irradiation time and power level of microwave set up are 7-12 min, 60 and 100\% power. The synthesized compounds were identified on the basis of IR, ${ }^{1} \mathrm{H}$ NMR, ${ }^{13} \mathrm{C}$ NMR, mass spectra and elemental analysis.

Table 2. Optimization for the reaction of fatty acid hydrazides with 1,2-diketones under microwave irradiation

\begin{tabular}{|c|c|c|c|c|c|c|c|c|}
\hline Entry & 1 & 2 & $\begin{array}{c}\text { Ratio } \\
1 / 2^{\mathrm{a}} \\
\end{array}$ & $\begin{array}{l}\text { Microwave } \\
\text { Equipment }\end{array}$ & $\begin{array}{c}\text { Power }^{\mathrm{c}} \\
(\%)\end{array}$ & $\begin{array}{c}\text { Time }^{\mathrm{d}} \\
\text { (minutes) }\end{array}$ & Product & $\begin{array}{c}\text { Yield }^{\mathrm{e}} \\
(\%)\end{array}$ \\
\hline 1 & $1 \mathbf{a}$ & $2 \mathbf{a}$ & $1: 1$ & Multimode & 60 & 7 & $3 \mathbf{a}$ & 92 \\
\hline 2 & 1b & $2 \mathbf{a}$ & $1: 1$ & Multimode & 60 & 7 & $3 \mathbf{b}$ & 92 \\
\hline 3 & 1c & $2 a$ & $1: 1$ & Multimode & 60 & 8 & $3 c$ & 92 \\
\hline 4 & 1d & $2 \mathbf{a}$ & $1: 1$ & Multimode & 100 & 8 & 3d & 89 \\
\hline 5 & 1e & $\mathbf{2 a}$ & $1: 1$ & Multimode & 100 & 8 & $3 e$ & 90 \\
\hline 6 & 1f & $\mathbf{2 a}$ & $1: 1$ & Multimode & 100 & 9 & $3 f$ & 89 \\
\hline 7 & $1 \mathrm{a}$ & $2 \mathbf{b}$ & $1: 1$ & Multimode & 60 & 7 & $3 g$ & 90 \\
\hline 8 & 1b & $2 \mathbf{b}$ & $1: 1$ & Multimode & 60 & 8 & $3 \mathbf{h}$ & 91 \\
\hline 9 & 1c & $2 \mathbf{b}$ & $1: 1$ & Multimode & 60 & 8 & $3 \mathbf{i}$ & 90 \\
\hline 10 & 1d & $2 \mathbf{b}$ & $1: 1$ & Multimode & 100 & 10 & $3 \mathbf{j}$ & 89 \\
\hline 11 & 1e & $2 \mathbf{b}$ & $1: 1$ & Multimode & 100 & 12 & $3 \mathbf{k}$ & 89 \\
\hline 12 & 1f & $2 \mathbf{b}$ & $1: 1$ & Multimode & 100 & 12 & 31 & 88 \\
\hline
\end{tabular}

${ }^{a}$ All reactions were carried out using fatty acid hydrazides (1 eq) with respect to 1,3-diketone under microwave irradiation. ${ }^{b}$ Microwave equipment multimode was used. ${ }^{c}$ Full power is 1.35 $\mathrm{kHz} .{ }^{\mathrm{d}}$ Monitored by TLC. ${ }^{\mathrm{e}}$ All yields refer to isolated products and the products were characterized by IR, ${ }^{1} \mathrm{H}$ NMR, ${ }^{13} \mathrm{C}$ NMR, MS and elemental analysis.

\section{Antimicrobial activity}

To check the biological activity of the compounds, the series of the compounds (3a-l) were screened for antimicrobial activity against bacteria (e.g. Escherichia coli, Staphylococcus aureus, Bacillus subtilis, Salmonella typhimurium), fungi (Penicillium, Helminthosporum oryzae, Aspergillus niger) and Candida albicans. The disc diffusion method with little modification was used. $^{40}$ Briefly, $0.1 \mathrm{ml}$ of diluted inoculum $\left(10^{5} \mathrm{CFU} / \mathrm{ml}\right)$ of test organism was spread on nutrient agar (NA) and sabaroud dextrose (SD) agar plates. Sterile filter paper disc (8 mm) (Hi-Media Pvt Ltd, Mumbai, India) impregnated with $50 \mu \mathrm{g}$ of the compound and a disc without compound was used as a negative control. The NA plates were incubated for $18 \mathrm{~h}$ at $37^{\circ} \mathrm{C}$ for test bacteria, $C$. 
albicans and SD plates for fungi were incubated for 5-6 days at $25{ }^{\circ} \mathrm{C}$. The activity was determined by measuring the zone of growth inhibition of the test organism. Antibiotics, chloramphenicol and nystatin (Hi-Media Pvt Ltd, Mumbai, India) were used in the test system as positive controls. The compounds were dissolved in DMF. The results are shown in Table 3. The compounds showed good activity against $H$. oryzae, $S$. aureus, E. coli and better results were obtained against $C$. albicans, Penicillium, A. niger. The moderate activity of compounds was observed against $S$. typhimurium and B.subtilis.

Table 3. Antimicrobial activity of 3,5,6-trisubstituted-1,2,4-triazines

\begin{tabular}{|c|c|c|c|c|c|c|c|c|}
\hline Entry & $\begin{array}{c}\text { E.coli } \\
(\mathrm{K} \\
12)\end{array}$ & $\begin{array}{c}\text { B. } \\
\text { subtilis } \\
\text { (ATCC } \\
\text { 6501) }\end{array}$ & $\begin{array}{c}S . \\
\text { aureus } \\
\text { (MSSA } \\
\text { 22) }\end{array}$ & $\begin{array}{c}\text { S.typhimurium } \\
\text { (МTCC 98) }\end{array}$ & $\begin{array}{l}\text { C.albicans } \\
\text { (IOA-109) }\end{array}$ & $\begin{array}{c}\text { Penicillium } \\
\text { Laboratory } \\
\text { isolate }\end{array}$ & $\begin{array}{c}A . \text { niger } \\
\text { Laboratory } \\
\text { isolate }\end{array}$ & $\begin{array}{c}\text { H.oryzae } \\
(2537)\end{array}$ \\
\hline $3 a$ & ++ & + & +++ & + & +++ & +++ & ++ & ++ \\
\hline $\mathbf{3 b}$ & ++ & + & +++ & + & +++ & +++ & +++ & +++ \\
\hline $3 c$ & ++ & ++ & ++ & + & +++ & +++ & +++ & +++ \\
\hline 3d & ++ & + & ++ & + & +++ & ++ & +++ & +++ \\
\hline $3 e$ & + & + & ++ & + & +++ & ++ & +++ & ++ \\
\hline $3 f$ & + & + & ++ & + & +++ & ++ & ++ & ++ \\
\hline $3 g$ & ++ & + & ++ & + & +++ & ++ & ++ & ++ \\
\hline $3 \mathrm{~h}$ & ++ & + & ++ & + & ++ & ++ & ++ & ++ \\
\hline $3 \mathbf{i}$ & +++ & + & ++ & + & ++ & +++ & ++ & ++ \\
\hline $\mathbf{3 j}$ & ++ & + & ++ & + & ++ & + & ++ & ++ \\
\hline $3 k$ & + & + & ++ & + & + & + & ++ & ++ \\
\hline 31 & ++ & + & ++ & + & + & + & ++ & ++ \\
\hline Control DMF & --- & --- & --- & --- & --- & --- & --- & --- \\
\hline Chloroamphenicol & +++ & +++ & +++ & +++ & --- & --- & --- & --- \\
\hline Nystatin & --- & --- & --- & --- & +++ & +++ & +++ & +++ \\
\hline
\end{tabular}

Zone of diameter of growth inhibition; $<10 \mathrm{~mm} \mathrm{(-),} \mathrm{10-12} \mathrm{mm}(+), 13-15 \mathrm{~mm}(++), 16-20 \mathrm{~mm}$ $(+++)$.

\section{Experimental Section}

General Procedures. (9Z,12R)-12-Hydroxyoctadec-9-enoic (ricinoleic) and (9R,12Z)-9hydroxyoctadec-12-enoic (isoricinoleic) acids were isolated from the natural sources i.e. from Ricinus communis and Wrightia tinctoria seed oils respectively. The concentrate of pure hydroxy acids were obtained by Gunstone's partitioning ${ }^{41}$ of freshly prepared fatty acids and further 
purified by column chromatography. The esters of fatty acids were prepared by refluxing the fatty acid in methanol in the presence of catalytic amount of sulfuric acid. General (GR) grade of solvents were employed for the extraction purposes and when required solvents were dried and distilled before use. Homogeneity of the product was observed on TLC. ${ }^{1} \mathrm{H}$ NMR spectra were recorded in $\mathrm{CDCl}_{3}$ on a Bruker DRX-400 instrument. The chemical shifts $(\delta)$ were measured relative to TMS as an internal standard. Coupling constants $(J)$ are expressed in Hz. Mass spectra were obtained on a Jeol SX-102 (FAB) spectrometer. IR spectra were obtained on Shimadzu 8201 PC FT-IR using KBr pellet with absorption given in $\mathrm{cm}^{-1}$. The microwave irradiations were carried out using an unmodified domestic oven (LG, Model MC-808WAR, 1.35 KW, $2450 \mathrm{MHz})$.

\section{General procedure for preparation of fatty acid hyrazides}

The hydrazides of the fatty acids were prepared by refluxing the methyl esters $(0.01 \mathrm{mmol})$ with hydrazine hydrate $(0.02 \mathrm{mmol})$ in methanol at about $130{ }^{\circ} \mathrm{C}$ for $2 \mathrm{~h}^{42}$ The mixture was then cooled to room temperature. The hydrazide solidified and was filtered, washed with water and recrystallized from ethanol. The fatty acid hydrazides were characterized by melting points and mixed melting points and spectral data.

\section{General procedure for synthesis of 3,5,6-trisubstituted-1,2,4-triazines}

A mixture of fatty acid hydrazide $(2 \mathrm{mmol})$, diketone $(2 \mathrm{mmol})$ and silica gel was ground in a pestle, $\mathrm{NH}_{4} \mathrm{OAc}$ and $\mathrm{Et}_{3} \mathrm{~N}$ were added in catalytic amounts and the prepared mixture in an open pyrex beaker was subjected to microwave irradiation for the appropriate time (Table 2). After complete conversion as indicated by TLC, the mixture was extracted with petroleum ether $(3 \times 50$ $\mathrm{ml})$ and washed with water $(3 \times 50 \mathrm{ml})$. Then the solvent was evaporated in vacuum and the product was purified by column chromatography.

Hexadecanoic acid hydrazide (1a). Yield $=95 \% ; \mathrm{Mp}=110-111^{\circ} \mathrm{C}$; IR (KBr): 3390, 3280, 2960, $2890 \mathrm{~cm}^{-1} ;{ }^{1} \mathrm{H}$ NMR (400 MHz, $\left.\mathrm{CDCl}_{3}\right): 8.10$ (s, 1H), 4.13 (d, J=6.9 Hz, 2H), 2.78 (t, J=7.9 $\mathrm{Hz}, 2 \mathrm{H}), 1.95$ (m, 2H), 1.30 (br.s, 24H), 0.88 (dist. t, 3H); MS: $270\left(\mathrm{M}^{+}\right.$), 157, 87, 73, 53. Anal: Calcd. for $\mathrm{C}_{16} \mathrm{H}_{34} \mathrm{ON}_{2}$ : C, 71.11; H, 12.59; N, 10.37\% Found: C, 71.09; H, 12.30; N, 10.61\%.

Octadecanoic acid hydrazide (1b). Yield $=92 \%$; $\mathrm{Mp}=112-114{ }^{\circ} \mathrm{C}$; IR (KBr): 3390, 3280, 2960, $2890 \mathrm{~cm}^{-1} ;{ }^{1} \mathrm{H}$ NMR (400 MHz, $\mathrm{CDCl}_{3}$ ): 8.10 (s, 1H), 4.13 (d, J=6.9 Hz, 2H), 2.77 (t, $J=7.9$ Hz, 2H), 1.92 (m, 2H), 1.30 (br.s ,28H), 0.88 (3H, dist. t, $\left.\mathrm{CH}_{3}\right)$; MS: $298\left(\mathrm{M}^{+}\right), 157$, 87, 73, 53. Anal: Calcd. for $\mathrm{C}_{18} \mathrm{H}_{38} \mathrm{ON}_{2}$ : C, 72.98; H, 12.75; N, 9.39\% Found: C, 72.55; H, 12.30; N, $9.43 \%$.

10-Undecenoic acid hydrazide (1c). Yield $=90 \% ; \mathrm{Mp}=78-80{ }^{\circ} \mathrm{C}$; IR (KBr): 3390, 3280, 2960, $2890 \mathrm{~cm}^{-1} ;{ }^{1} \mathrm{H} \mathrm{NMR}\left(400 \mathrm{MHz}, \mathrm{CDCl}_{3}\right): \delta 8.10(\mathrm{~s}, 1 \mathrm{H}), 5.82\left(\mathrm{tdd}, 1 \mathrm{H}, J_{\mathrm{H}-^{9} \mathrm{CH}_{2}}=6.6 \mathrm{~Hz}, J_{\mathrm{H}-\mathrm{H}_{Z}}=\right.$ $\left.10.2 \mathrm{~Hz}, J_{H-H_{E}}=17.1 \mathrm{~Hz}, \mathrm{CH}_{2}=\mathrm{CH}-\right), 5.02\left(1 \mathrm{H}, \mathrm{dd}, J_{H_{Z}-H}=10.2 \mathrm{~Hz}, J_{H_{Z}-H_{E}}=1.2 \mathrm{~Hz}\right.$, $\left.H_{Z} \mathrm{C}=\mathrm{CH}\right), 4.90\left(1 \mathrm{H}, \mathrm{dd}, J_{H_{E}-H}=17.1 \mathrm{~Hz}, J_{H_{E}-H_{Z}}=3.6 \mathrm{~Hz}, H_{E} \mathrm{C}=\mathrm{CH}-\right), 4.11(\mathrm{~d}, J=6.8 \mathrm{~Hz}$, 
2H), 2.88 (t, $J=7.9 \mathrm{~Hz}, 2 \mathrm{H}), 2.06(\mathrm{~m}, 2 \mathrm{H}), 1.89(\mathrm{~m}, 2 \mathrm{H}), 1.38$ (br.s, 10H); MS: $198\left(\mathrm{M}^{+}\right), 87$, 73, 59, 41. Anal: Calcd. for $\mathrm{C}_{11} \mathrm{H}_{22} \mathrm{ON}_{2}$ : C, 66.66; H, 11.11; N, 14.14\%. Found: C, 66.56; H, $11.09 ; \mathrm{N}, 14.12 \%$.

Octadec-9-enoic acid hydrazide (1d). Yield $=90 \% ; \mathrm{Mp}=110-112{ }^{\circ} \mathrm{C}$; IR (KBr): 3390, 3280, 2960, $2890 \mathrm{~cm}^{-1} ;{ }^{1} \mathrm{H}$ NMR (400 MHz, $\left.\mathrm{CDCl}_{3}\right): \delta 8.10(\mathrm{~s}, 1 \mathrm{H}), 5.34$ (m, 2H), 4.13 (d, J=6.9 Hz, 2H), 2.78 (t, $J=7.9 \mathrm{~Hz}, 2 \mathrm{H}), 2.02(\mathrm{~m}, 4 \mathrm{H}), 1.95$ (m, 2H), 1.30 (br.s, 20H), 0.88 (dist. t, 3H); MS: $293\left(\mathrm{M}^{+}\right), 157,87,73$, 53. Anal: Calcd. for $\mathrm{C}_{18} \mathrm{H}_{36} \mathrm{ON}_{2}$ : C, 73.32; H, 12.28; N, 9.55\% Found: C, 73.40; H, 12.30; N, 9.43\%.

(9Z,12R)-12-Hydroxyoctadec-9-enoic acid hydrazide (1e). Yield $85 \%$; Mp $=108-110^{\circ} \mathrm{C}$; IR (KBr): 3390, 3280, 2960, $2890 \mathrm{~cm}^{-1} ;{ }^{1} \mathrm{H}$ NMR (400 MHz, $\left.\mathrm{CDCl}_{3}\right): \delta 8.20(\mathrm{~s}, 1 \mathrm{H}), 5.46(\mathrm{~m}$, $2 \mathrm{H}), 3.88(\mathrm{~m}, 1 \mathrm{H}), 2.88(\mathrm{t}, J=7.9 \mathrm{~Hz}, 2 \mathrm{H}), 2.31(\mathrm{~m}, 1 \mathrm{H}), 4.16(\mathrm{~d}, J=6.9 \mathrm{~Hz}, 2 \mathrm{H}), 2.04(\mathrm{~m}, 4 \mathrm{H})$, 1.93 (m, 2H), 1.33 (br.s, 18H), 0.86 (dist.t, 3H); MS: $309\left(\mathrm{M}^{+}\right), 175,87,73$, 59. Anal: Calcd. for $\mathrm{C}_{18} \mathrm{H}_{36} \mathrm{O}_{2} \mathrm{~N}_{2}: \mathrm{C}, 69.90 ; \mathrm{H}, 11.65 ; \mathrm{N}, 9.06 \%$ Found: $\mathrm{C}, 69.88 ; \mathrm{H}, 11.62 ; \mathrm{N}, 9.08 \%$.

(9R,12Z)-9-Hydroxyoctadec-12-enoic acid hydrazide (1f). Yield 80\%; Mp $=110-112^{\circ} \mathrm{C}$; IR (KBr): 3390, 3280, 2960, $2890 \mathrm{~cm}^{-1} ;{ }^{1} \mathrm{H}$ NMR $\left(400 \mathrm{MHz}, \mathrm{CDCl}_{3}\right): \delta 8.11(\mathrm{~s}, 1 \mathrm{H}), 5.39(\mathrm{~m}$, 2H), 4.11 (d, $J=6.9 \mathrm{~Hz}, 2 \mathrm{H}), 3.76(\mathrm{~m}, 1 \mathrm{H}), 2.90$ (t, $J=7.9 \mathrm{~Hz}, 2 \mathrm{H}), 2.28(\mathrm{~m}, 1 \mathrm{H}), 2.09(\mathrm{~m}, 4 \mathrm{H})$, 1.98 (m, 2H), 1.31 (br.s, 18H), 0.86 (dist.t, 3H); MS: $309\left(\mathrm{M}^{+}\right), 199,87,73$, 59. Anal: Calcd. for $\mathrm{C}_{18} \mathrm{H}_{36} \mathrm{O}_{2} \mathrm{~N}_{2}$ : C, 69.90; H, 11.65; N, 9.06\% Found: C, 69.88; H, 11.62; N, 9.08\%.

3-Pentadecyl-5,6-dimethyl-1,2,4-triazine (3a). IR (KBr): 2930, 2859, 1594, $778 \mathrm{~cm}^{-1}$; ${ }^{1} \mathrm{H}$ NMR (400 MHz, $\mathrm{CDCl}_{3}$ ): $\delta 2.88$ (t, $\left.J=6.84 \mathrm{~Hz}, 2 \mathrm{H}\right), 2.35$ (m, 6H), 1.62 (m, 2H), 1.28 (br.s. 24H). 0.88 (dist.t. $3 \mathrm{H}) ;{ }^{13} \mathrm{C}$ NMR $\left(100 \mathrm{MHz}, \mathrm{CDCl}_{3}\right): \delta 178.71,176.77,174.62,34.62,33.99,31.64,29.32$, 28.01, 25.47, 23.42, 14.17; MS: $319\left(\mathrm{M}^{+}\right), 150,136,122$, 108. Anal: Calcd. for $\mathrm{C}_{20} \mathrm{H}_{37} \mathrm{~N}_{3}: \mathrm{C}$, 75.23; H, 11.59; N, 13.16\% Found: C, 75.16; H, 11.60; N, 13.20\%

3-Heptadecyl-5,6-dimethyl -1,2,4-triazine (3b). IR (KBr): 2930, 2860, 1590, $770 \mathrm{~cm}^{-1} ;{ }^{1} \mathrm{H}$ NMR (400 MHz, $\left.\mathrm{CDCl}_{3}\right): \delta 2.80(\mathrm{t}, J=6.80 \mathrm{~Hz}, 2 \mathrm{H}), 2.35(\mathrm{~m}, 6 \mathrm{H}), 1.63(\mathrm{~m}, 2 \mathrm{H}), 1.28$ (br.s. 28H). 0.88 (dist.t. $3 \mathrm{H}) ;{ }^{13} \mathrm{C}$ NMR (100 MHz, $\left.\mathrm{CDCl}_{3}\right): \delta 180.10,179.41,177.09,34.09,31.95$, 29.42, 24.71, 22.72, 14.15; MS: $347\left(\mathrm{M}^{+}\right), 330,232,122$, 108. Anal: Calcd. for $\mathrm{C}_{22} \mathrm{H}_{41} \mathrm{~N}_{3}: \mathrm{C}$, 76.08; H, 12.10; N, 11.81\% Found: C, 76.11; H, 12.13; N, 11.89\%

3-(Dec-9-enyl)-5,6-dimethyl-1,2,4-triazine (3c). IR (KBr): 2928, 2866, 1580, $775 \mathrm{~cm}^{-1} ;{ }^{1} \mathrm{H}$ $\operatorname{NMR}\left(400 \mathrm{MHz}, \mathrm{CDCl}_{3}\right): \delta 5.82\left(1 \mathrm{H}\right.$, tdd, $J_{H_{-}^{-9} \mathrm{CH}_{2}}=6.6 \mathrm{~Hz}, J_{H-H_{Z}}=10.2 \mathrm{~Hz}, J_{H-H_{E}}=17.1 \mathrm{~Hz}$, $\left.\mathrm{CH}_{2}=\mathrm{C} H-\right), 5.02\left(1 \mathrm{H}, \mathrm{dd}, J_{H_{Z}-H}=10.2 \mathrm{~Hz}, J_{H_{Z}-H_{E}}=1.2 \mathrm{~Hz}, H_{Z} \mathrm{C}=\mathrm{CH}-\right), 4.90\left(1 \mathrm{H}, \mathrm{dd}, J_{H_{E}-H}=\right.$ $\left.17.1 \mathrm{~Hz}, J_{H_{E}-H_{Z}}=1.2 \mathrm{~Hz}, H_{E} \mathrm{C}=\mathrm{CH}-\right), 2.80(\mathrm{t}, J=6.8 \mathrm{~Hz}, 2 \mathrm{H}), 2.36(\mathrm{~m}, 6 \mathrm{H}) 2.03(\mathrm{~m}, 2 \mathrm{H}), 1.63$ (m, 2H), 1.29 (br.s. $10 \mathrm{H}) ;{ }^{13} \mathrm{C}$ NMR $\left(100 \mathrm{MHz}, \mathrm{CDCl}_{3}\right): \delta 180.64,179.76,178.45,139.19$, 114.22, 34.02, 29.15, 24.72; MS: $247\left(\mathrm{M}^{+}\right), 220,139,108$. Anal: Calcd. for $\mathrm{C}_{15} \mathrm{H}_{25} \mathrm{~N}_{3}$ : C, 72.87 ; H, 10.12; N, 17.00\% Found: C, 72.99; H, 10.16; N, 17.12\%

3-(Heptadec-8-enyl)-5,6-dimethyl-1,2,4-triazine (3d). IR (KBr): 2930, 2856, 1585, $775 \mathrm{~cm}^{-1}$; ${ }^{1} \mathrm{H}$ NMR $\left(400 \mathrm{MHz}, \mathrm{CDCl}_{3}\right): \delta 5.35(\mathrm{~m}, 2 \mathrm{H}), 2.77(\mathrm{t}, J=6.52 \mathrm{~Hz}, 2 \mathrm{H}), 2.34(\mathrm{~m}, 6 \mathrm{H}), 2.03(\mathrm{~m}$, 4H), 1.63 (m, 2H), 1.30 (br.s, $18 \mathrm{H}$ ), 0.88 (dist. t. $3 \mathrm{H}) ;{ }^{13} \mathrm{C} \mathrm{NMR} \mathrm{(100} \mathrm{MHz,} \mathrm{CDCl}_{3}$ ): $\delta 179.76$, 176.77, 174.37, 130.01, 129.72, 44.37, 34.00, 31.64, 29.39, 27.13, 25.54, 24.74, 14.17; MS: 345 
$\left(\mathrm{M}^{+}\right)$, 330, 232, 122, 108; Anal: Calcd. for $\mathrm{C}_{22} \mathrm{H}_{39} \mathrm{~N}_{3}$ : C, 76.52; H, 11.30; N, 12.17\% Found: C, 76.50; H, 11.33; N, 12.19\%

3-[(8Z, 11R)-11-Hydroxyheptadec-8-enyl]-5,6-dimethyl-1,2,4-triazine (3e). IR (KBr): 2940, 2866, 1580, $778 \mathrm{~cm}^{-1}$; ${ }^{1} \mathrm{H}$ NMR (400 MHz, $\left.\mathrm{CDCl}_{3}\right): \delta 5.38(\mathrm{~m}, 2 \mathrm{H}), 3.64(\mathrm{~m}, 1 \mathrm{H}), 2.88(\mathrm{t}, J=6.82$ Hz, 2H), 2.30 (m, 1H), $2.21(\mathrm{~m}, 6 \mathrm{H}), 2.03(\mathrm{~m}, 4 \mathrm{H}), 1.54$ (m, 2H), 1.37 (br.s, 18H), 0.88 (dist.t. $3 \mathrm{H}) ;{ }^{13} \mathrm{C} \mathrm{NMR}\left(100 \mathrm{MHz}, \mathrm{CDCl}_{3}\right): \delta 178.73,177.00,175.02,127.98,127.83,77.67,37.05$, 34.63, 32.41, 29.16, 24.29, 14.15; MS: $361\left(\mathrm{M}^{+}\right), 276,136,122$, 108; Anal: Calcd. for $\mathrm{C}_{22} \mathrm{H}_{39}$ $\mathrm{ON}_{3}$ : C, 73.13; H, 11.63; N, 10.80\% Found: C, 73.11; H, 11.70; N, 10.89\%

3-[(8R, 11Z)-8-Hydroxyheptadec-11-enyl]-5,6-dimethyl-1,2,4-triazine (3f). IR (KBr): 2930, 2859, 1594, $778 \mathrm{~cm}^{-1} ;{ }^{1} \mathrm{H}$ NMR (400 MHz, $\left.\mathrm{CDCl}_{3}\right): \delta 5.38(\mathrm{~m}, 2 \mathrm{H}), 3.64(\mathrm{~m}, 1 \mathrm{H}), 2.83(\mathrm{t}, J=6.82$ $\mathrm{Hz}, 2 \mathrm{H}), 2.24(\mathrm{~m}, 6 \mathrm{H}), 2.04(\mathrm{~m}, 4 \mathrm{H}), 1.63$ (m, 2H), 1.36 (br.s, 18H), 0.88 (dist. t. $3 \mathrm{H}) ;{ }^{13} \mathrm{C} \mathrm{NMR}$ $\left(100 \mathrm{MHz}, \mathrm{CDCl}_{3}\right): \delta 178.71,176.77,174.62,130.04,129.67,127.86,71.67,37.06,34.60$, 33.98, 31.64, 29.28, 24.29, 14.50; MS: $361\left(\mathrm{M}^{+}\right)$, 236, 136, 122, 108. Anal: Calcd. for $\mathrm{C}_{22} \mathrm{H}_{39} \mathrm{ON}_{3}$ : C, 73.13; H, 11.63; N, 10.80\% Found: C, 73.19; H, 11.80; N, 10.99\%.

3-Pentadecyl-5,6-diphenyl-1,2,4-triazine (3g). IR (KBr): 2930, 1590, 2860, $770 \mathrm{~cm}^{-1}$; ${ }^{1} \mathrm{H}$ NMR $\left(300 \mathrm{MHz}, \mathrm{CDCl}_{3}\right): \delta$ 7.53-7.26 (10 H, m, Ar-H), 2.52 (t, J=7.2Hz, 2H), $1.96(\mathrm{~m}, 2 \mathrm{H}), 1.47$ (br.s, $24 \mathrm{H}), 0.88$ (dist. t. $3 \mathrm{H}) ;{ }^{13} \mathrm{C}$ NMR $\left(100 \mathrm{MHz}, \mathrm{CDCl}_{3}\right): \delta 180.33,179.76,178.45,134.94,133.04$, 129.51, 34.15, 31.98, 29.45, 23.74, 14.17; MS: $443\left(\mathrm{M}^{+}\right)$, 428, 260, 246, 232. Anal: Calcd. for $\mathrm{C}_{30} \mathrm{H}_{41} \mathrm{~N}_{3}$ : C, 81.26; H, 9.25; N, 9.48\% Found: C, 81.30; H, 11.70; N, 9.09\%

3-Heptadecyl-5,6-diphenyl-1,2,4-triazine (3h). IR (KBr): 2930, 2859, 1594, $778 \mathrm{~cm}^{-1}$; ${ }^{1} \mathrm{H}$ NMR (300 MHz, $\left.\mathrm{CDCl}_{3}\right): \delta 7.53-7.26(10 \mathrm{H}, \mathrm{m}, \mathrm{Ar}-\mathrm{H}), 2.52(\mathrm{t}, 2 \mathrm{H}, J=6.9 H z, 2 \mathrm{H}), 1.96(\mathrm{~m}, 2 \mathrm{H})$, 1.47 (br.s, 28H), 0.88 (dist. t. 3H); ${ }^{13} \mathrm{C} \mathrm{NMR} \mathrm{(100} \mathrm{MHz,} \mathrm{CDCl}_{3}$ ): $\delta$ 180.10, 179.76, 173.83, 134.92, 133.01, 129.49, 34.09, 31.95, 29.42, 23.71, 14.15; MS: $471\left(\mathrm{M}^{+}\right), 456,260,246,232$; Anal: Calcd. for $\mathrm{C}_{32} \mathrm{H}_{45} \mathrm{~N}_{3}$ : C, 81.52; H, 9.55; N, 8.91\% Found: C, 81.30; H, 9.66; N, 8.70\% 3-(Dec-9-enyl)-5,6-diphenyl-1,2,4-triazine (3i). IR (KBr): 2933, 2856, 1580, $776 \mathrm{~cm}^{-1}$; ${ }^{1} \mathrm{H}$ NMR (300 MHz, $\left.\mathrm{CDCl}_{3}\right): \delta 8.03-7.25(\mathrm{~m}, 10 \mathrm{H}), 5.82\left(1 \mathrm{H}, \mathrm{tdd}, J_{\mathrm{H}^{9}{ }^{9} \mathrm{CH}_{2}}=6.6 \mathrm{~Hz}, J_{\mathrm{H}_{-} \mathrm{H}_{\mathrm{Z}}}=10.2\right.$ $\left.\mathrm{Hz}, J_{H-H_{E}}=17.1 \mathrm{~Hz}, \mathrm{CH}_{2}=\mathrm{CH}-\right), 5.02\left(1 \mathrm{H}, \mathrm{dd}, J_{H_{Z}-H}=10.2 \mathrm{~Hz}, J_{H_{Z}-H_{E}}=1.2 \mathrm{~Hz}, H_{Z} \mathrm{C}=\mathrm{CH}-\right)$, $4.90\left(1 \mathrm{H}, \mathrm{dd}, J_{H_{E}-H}=17.1 \mathrm{~Hz}, J_{H_{E}-H_{Z}}=1.2 \mathrm{~Hz}, H_{E} \mathrm{C}=\mathrm{CH}-\right), 2.81(\mathrm{t}, J=7.6 \mathrm{~Hz}, 2 \mathrm{H}), 2.02(\mathrm{~m}$, 2H), 1.68 (m, 2H), 1.29 (br.s. 10H); MS: $373\left(\mathrm{M}^{+}\right), 346,260,246,232 .{ }^{13} \mathrm{C} \mathrm{NMR}(100 \mathrm{MHz}$, $\left.\mathrm{CDCl}_{3}\right): \delta 178.71,176.77,174.62,130.56,130.47,128.78,34.62,33.99,31.64,28.88,24.30$; Anal: Calcd. for $\mathrm{C}_{25} \mathrm{H}_{29} \mathrm{~N}_{3}$ : C, 80.42; H, 8.31; N, 11.26\% Found: C, 80.36; H, 8.39; N, 11.22\%

3-(Heptadec-8-enyl)-5,6-diphenyl-1,2,4-triazine (3j). IR (KBr): 2933, 2855, 1580, 770 cm$^{-1}$; ${ }^{1} \mathrm{H}$ NMR (300 MHz, $\left.\mathrm{CDCl}_{3}\right): \delta 7.85-7.33(\mathrm{~m}, 10 \mathrm{H}), 5.35(\mathrm{~m}, 2 \mathrm{H}), 2.79(\mathrm{t}, J=7.2 \mathrm{~Hz}, 3 \mathrm{H}), 2.02$ $(\mathrm{m}, 4 \mathrm{H}), 1.73(\mathrm{~m}, 2 \mathrm{H}), 1.26$ (br.s, 20H), 0.88 (dist.t. $3 \mathrm{H}) ;{ }^{13} \mathrm{C} \mathrm{NMR}\left(100 \mathrm{MHz}, \mathrm{CDCl}_{3}\right): \delta$ 180.33 , 178.95, 176.77, 134.94, 133.04, 129.51, 34.15, 31.98, 29.45, 23.74, 14.17; MS: 469 $\left(\mathrm{M}^{+}\right)$, 356, 260, 246, 232. Anal: Calcd. for $\mathrm{C}_{32} \mathrm{H}_{43} \mathrm{~N}_{3}$ : C, 81.87; H, 9.16; N, 8.95\% Found: C, 81.36; H, 9.39; N, 8.82\%

3-[(8Z, 11R)-11-Hydroxyheptadec-8-enyl]-5,6-diphenyl-1,2,4-triazine (3k). IR (KBr): 2930, 2850, 1585, $776 \mathrm{~cm}^{-1} ;{ }^{1} \mathrm{H}$ NMR (300 MHz, $\left.\mathrm{CDCl}_{3}\right): \delta 8.03-7.21(\mathrm{~m}, 10 \mathrm{H}), 5.36(\mathrm{~m}, 2 \mathrm{H}), 3.89$ 
(m, 1H), $2.04(\mathrm{~m}, 4 \mathrm{H}), 2.61(\mathrm{t}, J=7.5 \mathrm{~Hz}, 2 \mathrm{H}), 2.27(\mathrm{~m}, 1 \mathrm{H}), 2.04(\mathrm{~m}, 4 \mathrm{H}), 1.51(\mathrm{~m}, 2 \mathrm{H}), 1.25$ (br.s. $18 \mathrm{H}), 0.86$ (dist. t. $3 \mathrm{H}) ;{ }^{13} \mathrm{C} \mathrm{NMR}\left(100 \mathrm{MHz}, \mathrm{CDCl}_{3}\right): \delta 178.71,177.09,175.06,130.56$, 130.47, 128.78, 71.67, 37.06, 34.30, 31.64, 28.88, 24.30, 14.15; MS: $485\left(\mathrm{M}^{+}\right), 400,260,246$, 232. Anal: Calcd. for $\mathrm{C}_{32} \mathrm{H}_{43} \mathrm{ON}_{3}$ : C, 79.17; H, 8.86; N, 8.65\% Found: C, 79.20; H, 8.89; N, 8.60 3-[(8R, 11Z)-8-Hydroxyheptadec-11-enyl]-5,6-diphenyl-1,2,4-triazine (3I). IR (KBr): 2940, 2866, 1585, $778 \mathrm{~cm}^{-1} ;{ }^{1} \mathrm{H}$ NMR (300 MHz, $\left.\mathrm{CDCl}_{3}\right): \delta 7.35-7.00(\mathrm{~m}, 10 \mathrm{H}), 5.46(\mathrm{~m}, 2 \mathrm{H}), 3.91$ (m, 1H), $2.54(\mathrm{t}, J=7.5 \mathrm{~Hz}, 2 \mathrm{H}), 2.36(\mathrm{~m}, 1 \mathrm{H}), 2.02(\mathrm{~m}, 4 \mathrm{H}), 1.73(\mathrm{~m}, 2 \mathrm{H}), 1.38$ (br.s. $18 \mathrm{H}), 0.88$ (dist. t. $3 \mathrm{H}) ;{ }^{13} \mathrm{C} \mathrm{NMR}\left(100 \mathrm{MHz}, \mathrm{CDCl}_{3}\right): \delta 178.71,176.77,174.62,130.56,130.47,128.78$, 71.67, 37.06, 34.60, 33.96, 31.68, 28.90, 24.29, 14.50; MS: $485\left(\mathrm{M}^{+}\right)$, 360, 260, 246, 232. Anal: Calcd. for $\mathrm{C}_{32} \mathrm{H}_{43} \mathrm{ON}_{3}$ : C, 79.17; H, 8.86; N, 8.65\% Found: C, 79.16; H, 8.90; N, 8.59\%

\section{Conclusions}

The preparation of. 3,5,6-trisubstituted-1,2,4-triazines derivatives of fatty acid hydrazides is a valuable addition to the synthesis and manipulation of fatty acid derivatives. This work could be extended to some naturally occurring unusual fatty acids. Biological evaluation of these derivatives may furnish some other important applications.

\section{Acknowledgements}

The authors are grateful to the Chairman, Department of Chemistry, Aligarh Muslim University for providing the necessary research facilities. We wish to give special thanks to Dr. Iqbal Ahmad, Department of Agricultural Microbiology, AMU for antimicrobial activity of our compounds. We are also grateful to the SAIF, CDRI Lucknow and Panjab University for providing IR, ${ }^{1} \mathrm{H}$ NMR, ${ }^{13} \mathrm{C}$ NMR, mass spectra and elemental analysis.

\section{References}

1. (a) Kappe, C. O.; Dallinger, D. Nat. Rev. Drug Discovery 2006, 5, 55. (b) Shipe, W. D.; Wolkenberg, S. E.; Lindsley, C. W. Drug Discovery Today Tech 2005, 2, 155 . (c) Leadbeater, N. E. Chem. Commun. 2005, 2881.

2. Caddick, S. Tetrahedron. 1995, 51, 10403.

3. Kidwai, M.; Sapra, P.; Bhushan, K. R.; Saxena R. K.; Gupta, R.; Singh, M. Montash. Chem. 2000, 131, 85 .

4. Hermkens , P.; Ottenheijm, H.; Rees, D. Tetrahedron. 1997, 53, 5643.

5. Villemin, D.; Alloumn, A. B. Synth. Commun. 1991, 21, 63.

6. Loupy, A.; Pegion, P.; Ramdani, M.; Jacquault, P. Synth. Commun. 1994, 24 , 1159. 
7. Kidwai, M.; Sapra, P. Synth. Commun. 1999, 29, 3237.

8. (a) Geske, G. D.; Wezeman, R. J.; Siegel, A. P.; Blackwell, H. E. J. Am. Chem. Soc. 2005, 127, 12762. (b) Liu, J. -F.; Ye, P.; Sprague, K.; Sargent, K.; Yohannes, D.; Baldino, C. M.; Wilson, C. J.; Ng, S.- C. Org. Lett. 2005, 7, 3363. (c) Liu, J. -F.; Kaselj, M.; Isome, Y.; Chapnick, J.; Zhang, B.; Bi, G.; Yohannes, D.; Yu, L.; Baldino, C. M. J. Org. Chem. 2005, $70,10488$.

9. Boger, D. L. Chem. Rev. 1986, 86, 781.

10. Boger, D. L. Tetrahedron. 1983, 39, 2869.

11. Romanchick, W. A.; Joullie, M. M. Heterocycles. 1978, 9, 1631.

12. Neunhoeffer, H. Comprehensive Heterocyclic Chemistry, Katritzky, A. R. and Rees, C. W.; Eds Pergamon Press; Oxford 1984, 385.

13. Monge, A.; Palop, J.; Ramizrez, C.; Font, F. A. E. Eur. J. Med. Chem. 1991, 26, 83.

14. Labouta, I. M.; Eshba, N. H.; Salama, H. M. Farm. Sci. Ed 1998, 16, 29.

15. Abd. El-Samii, K. J. Chem. Tech. Biotech. 1992, 53, 143.

16. Leach, M. J.; Nobls, M. S. Eur. Pat. Appl. EP459, 829; Chem Abstr. 1992, 116, 128970x.

17. March. L. C.; Bajwa, G. S.; Lee, J.; Wasti, K.; Joullie, M. M. J. Med. Chem. 1976, 19, 845.

18. El-Gendy, Z.; Morsy, J.; Allimony, H.; Abdel-Monem, Abdel, Rehman, R. Phosphorous, Sulphur Silicon and the related Elements. 2003, 178, 2055.

19. Wieczorek, J.; Modarski, M.; Rykowski, A.; Nantka-Nimrski, P. Arch Immunol Ther Exp (Warsz) 1980, 28, 727.

20. Trepanier, D. L.; Wagner, E. R.; Harris, G.; Allan, D. J. Med. Chem. 1966, 9, 881.

21. Srinivas, K.; Srinivas, U.; Jayathirtha, Rao.; Bhanuprakash, K.; Harakishore, K.; Murthy, U.S.N. Bioorg. Med. Chem. Lett. 2005, 15, 1121.

22. Rusinov, V. L.; Ulomskii, E. N.; Chupakhin, O. N.; Zubairnov, M. M.; Kapustin, A. B.; Mitin, n. I.; Zhiravetskii, M. I.; Vinograd, I. A. Pharmaceut. Chem. J. 1990, 24, 640.

23. Gupta, A. S.; Bhattacharya, T.; Hajela, K.; Kripa Shankar, Ahmad, S. Pesticide Science, 1985, 16, 65 .

24. Smagin, S. S.; Bogachev, V. E.; Yakubovskii, A. K.; Metkalova, S. E.; Privol'neva, T. P.; Chugunon, V. V.; Lavretskaya, E. F. Pharmaceut. Chem. J. 1975, 9, 222.

25. Rubat, C.; Coudert, P.; Marvel, S.; Fialip, J.; Couquelet, J. J. Pharm. Pharmacol. 1999, 49, 1019.

26. Laasko, P. V., Robinson, R.; Vanderwala, H. P. Tetrahedron. 1957, 1, 103.

27. Metze, R. Chem. Ber. 1955, 88, 772.

28. (a) Metze, R. Chem. Ber. 1956, 89, 2058. (b) Metze, R.; Meyer, S. Chem. Ber. 1957, 90, 481. (c) Metze, R.; Kort, W. Chem. Ber. 1958, 91, 417.

29. Metze, R. Chem. Ber. 1954, 87, 1540.

30. Saraswathi, T. V.; Srinivasan, V. R. Tetrahedron Lett. 1971, 12, 2315.

31. Saraswathi, T. V.; Srinivasan, V. R. Tetrahedron 1977, 33, 1043.

32. Pauddler, W. W.; Barton, J. J. Org. Chem. 1966, 31, 1720. 
33. Neuhoeffer, V. H.; Henning, H.; Fruhauf, H. W.; Mutterer, M. Tetrahedron Lett. 1969, 10, 3147.

34. Ohsumi, T.; Neuhoeffer, H. Heterocycles. 1992, 33, 893.

35. (a) Rostamizadeh, R.; Sadeghi, K. Synth. Commun. 2002, 32, 1899. (b) Lindsley, C. W.; Wisnoski, D. D.; Wang, Yi.; Leister, W. H.;Zhao, Z. Tetrahedron Lett., 2003, 44, 4495. (c) Zhao, Z.; Leister, W. H.; Strauss, K. A.Wisnoski, D. D.; Lindsley, C. W. Tetrahedron Lett. 2003, 44, 1123.

36. Mustafa, J.; Ahmad Jr, M. S.; Rauf, A.; Osman, S.M. J. Am. Oil. Chem. Soc. 1984, 61, 555.

37. Rauf, A.; Parveen, H. J. Oleo. Sci. 2004, 53, 279.

38. Agrawal, R.; Rauf, A.; Ahmad, M. J. Am. Oil. Chem. Soc. 1989, 66, 970.

39. (a) Saeed, M. T.; Mustafa, J.; Rauf, A.; Osman, S.M. J. Am. Oil. Chem. Soc. 1992, 69, 481.

(b) Saeed, M.T.; Mustafa, J.; Rauf, A.; Osman, S.M. J. Am. Oil. Chem. Soc. 1992, 69, 396.

(c) Ansari, M.H.; Saeed, M.T.; Ahmad, M. 1990, J. Chem. Res. Synop, 1990, 126. (d) Rauf, A.; Ahmad, S. J. Chem. Res. 2005, 407.

40. Bauer, A. W.; Kirby W. M. M.; Sherris, J. C.; Turch, M. Am. J. Clin. Path. 1966, 45, 494.

41. Gunstone, F. D., J. Chem. Soc. 1954, 1611.

42. Markley, K. S. Fatty Acids their Chemistry, Properties, Production and Uses. Interscience Publishers, John Wiley and Sons: New York, 1964; Part 3. 